\title{
Wavelet estimation of a density in a GARCH-type model
}

\author{
Christophe Chesneau
}

Received:

\begin{abstract}
We consider the GARCH-type model: $S=\sigma^{2} Z$, where $\sigma^{2}$ and $Z$ are independent random variables. The density of $\sigma^{2}$ is unknown whereas the one of $Z$ is known. We want to estimate the density of $\sigma^{2}$ from $n$ observations of $S$ under some dependence assumption (the exponentially strongly mixing dependence). Adopting the wavelet methodology, we construct a nonadaptive estimator based on projections and an adaptive estimator based on the hard thresholding rule. Taking the mean integrated squared error over Besov balls, we prove that the adaptive one attains a sharp rate of convergence.
\end{abstract}

Keywords GARCH models · Adaptive density estimation · Rate of convergence · Wavelets · Hard thresholding.

2000 Mathematics Subject Classification 62G07, 62G20.

\section{Motivations}

We consider the random sequence $\left(S_{i}\right)_{i \in \mathbb{Z}}$ where, for any $i \in \mathbb{Z}$,

$$
S_{i}=\sigma_{i}^{2} Z_{i}
$$

$\left(Z_{i}\right)_{i \in \mathbb{Z}}$ is a sequence of identically distributed random variables with common known density $f_{Z}:[0,1] \rightarrow(0, \infty)$ and $\left(\sigma_{i}^{2}\right)_{i \in \mathbb{Z}}$ is a sequence of identically distributed random variables with common unknown density $f_{\sigma^{2}}:[0,1] \rightarrow(0, \infty)$. For any $i \in \mathbb{Z}, Z_{i}$ and $\sigma_{i}^{2}$ are independent. We suppose that $\left(S_{i}\right)_{i \in \mathbb{Z}}$ is strictly stationary and exponentially strongly mixing (to be defined in Section 2). We aim to estimate $f_{\sigma^{2}}$ when only $n$ random variables $S_{1}, \ldots, S_{n}$ are observed. The model (1) belongs to the family of the GARCH-type time series models classically encountered in financial models when the volatility process $\left(\sigma_{i}^{2}\right)_{i \in \mathbb{Z}}$ is unobserved (see for instance Carrasco and Chen (2002) for an overview).

Laboratoire de Mathématiques Nicolas Oresme, Université de Caen Basse-Normandie, Campus II, Science 3, 14032 Caen, France. E-mail: chesneau@math.unicaen.fr 
In the literature, the most common approach is to rewrite (1) as a convolution model via the logarithmic transformation: $\ln S_{i}=\ln \sigma_{i}^{2}+\ln Z_{i}, i \in \mathbb{Z}$. Then the density of $\ln \sigma_{1}^{2}$ is deconvolved and estimated by using Fourier transform and nonparametric methods (kernel, splines, wavelets, ....). See e.g. Masry (1991), Comte et al. (2008) and Van Zanten and Zareba (2008). In the independent case, methods ans results on the classical density convolution model can be found in Caroll and Hall (1988), Devroye (1989), Fan (1991), Pensky and Vidakovic (1999), Fan and Koo (2002), Butucea and Matias (2005), Comte et al. (2006), Delaigle and Gijbels (2006) and Lacour (2006). However, note that the density of $\ln \sigma_{1}^{2}$ is obviously not $f_{\sigma^{2}}$ and the estimation of $f_{\sigma^{2}}$ from $\ln S_{1}, \ldots, \ln S_{n}$ seems not really natural (see for instance (Comte et al. 2008, $3.5)$ ).

In this study, the "direct" estimation of $f_{\sigma^{2}}$ from $S_{1}, \ldots, S_{n}$ is investigated. Adopting a methodology similar to the one of Chaubey et al. (2010a) (for the density estimation problem under multiplicative censoring), we construct two new estimators based on wavelet basis. The first one, linear and nonadaptive, uses projections, and the second one, non-linear and adaptive, uses the hard thresholding rule introduced by Donoho et al. (1996). We evaluate their performances by determining upper bounds of the mean integrated squared error (MISE) over a wide range of smoothness function classes: the Besov balls. We prove that our adaptive estimator attains a sharp rate of convergence. "Sharp" in the sense that it is close to the one attained by the linear wavelet estimator constructed to minimize the MISE.

The paper is organized as follows. Assumptions on the model and some notations are introduced in Section 2. Section 3 briefly describes the wavelet basis and the Besov balls. The estimators are presented in Section 4. The results are set in Section 5. Technical proofs are collected in Section 6 .

\section{Assumptions and notations}

Let us now clarify the assumptions made on (1).

Assumption on $\left(S_{i}\right)_{i \in \mathbb{Z}}$. For any $m \in \mathbb{Z}$, we define the $m$-th strongly mixing coefficient of $\left(S_{i}\right)_{i \in \mathbb{Z}}$ by

$$
a_{m}=\sup _{(A, B) \in \mathcal{F}_{-\infty, 0}^{S} \times \mathcal{F}_{m, \infty}^{S}}|\mathbb{P}(A \cap B)-\mathbb{P}(A) \mathbb{P}(B)|
$$

where, for any $u \in \mathbb{Z}, \mathcal{F}_{-\infty, u}^{S}$ is the $\sigma$-algebra generated by the random variables $\ldots, S_{u-1}, S_{u}$ and $\mathcal{F}_{u, \infty}^{Y}$ is the $\sigma$-algebra generated by the random variables $S_{u}, S_{u+1}, \ldots$.

We consider the exponentially strongly mixing case i.e. there exist three known constants, $\gamma>0, c>0$ and $\theta>0$, such that, for any $m \in \mathbb{Z}$,

$$
a_{m} \leq \gamma \exp \left(-c|m|^{\theta}\right)
$$


This assumption is satisfied by a large class of GARCH processes. See e.g. Withers (1981), Doukhan (1994), Modha and Masry (1996) and Carrasco and Chen (2002).

Note that, when $\theta \rightarrow \infty$, we are in the standard i.i.d. case. So (1) becomes the classical density convolution model.

Assumption of $f_{Z}$. We suppose that there exists a positive integer $\nu$ such that

$$
f_{Z}(x)=\frac{1}{(\nu-1) !}(-\ln x)^{\nu-1}, \quad x \in[0,1]
$$

Note that $f_{Z}$ is the density of $\prod_{i=1}^{\nu} U_{i}$, where $U_{1}, \ldots, U_{n}$ are $n$ i.i.d. random variables having the common uniform distribution on $[0,1]$.

Assumption on $f_{\sigma^{2}}$. We assume w.o.l.g. that the support of $f_{\sigma^{2}}$ is $[0,1]$ and $f_{\sigma^{2}} \in \mathbb{L}^{2}([0,1])$, where

$$
\mathbb{L}^{2}([0,1])=\left\{h:[0,1] \rightarrow \mathbb{R} ; \quad\left(\int_{0}^{1} h^{2}(x) d x\right)^{1 / 2}<\infty\right\} .
$$

Assumption on the density of $S_{1}$. Note that, thanks to the independence of $\sigma_{1}^{2}$ and $Z_{1}$, the density of $S_{1}$ is

$$
f_{S}(x)=\int_{x}^{1} f_{Z}\left(\frac{x}{y}\right) f_{\sigma^{2}}(y) \frac{1}{y} d y, \quad x \in[0,1] .
$$

We suppose that there exists a known constant $C_{*}>0$ such that

$$
\sup _{x \in[0,1]} f_{S}(x) \leq C_{*}
$$

\section{Wavelets and Besov balls}

Wavelet basis. Let $N$ be an integer such that $N>\nu$ (where $\nu$ is the one in (3)), $\phi$ and $\psi$ be the initial wavelets of $d b N$. In particular, we have $-\operatorname{supp}(\phi)=\operatorname{supp}(\psi)=[1-N, N]$,

- $\phi$ and $\psi$ are of class $\mathcal{C}^{\nu}$.

Set

$$
\phi_{j, k}(x)=2^{j / 2} \phi\left(2^{j} x-k\right), \quad \psi_{j, k}(x)=2^{j / 2} \psi\left(2^{j} x-k\right) .
$$

With an appropriate treatments at the boundaries, there exists an integer $\tau$ satisfying $2^{\tau} \geq 2 N$ such that the collection $\mathcal{B}=\left\{\phi_{\tau, k}(),. k \in\left\{0, \ldots, 2^{\tau}-\right.\right.$ $\left.1\} ; \psi_{j, k}(.) ; j \in \mathbb{N}-\{0, \ldots, \tau-1\}, k \in\left\{0, \ldots, 2^{j}-1\right\}\right\}$, is an orthonormal basis of $\mathbb{L}^{2}([0,1])$. We refer to Cohen et al. (1993).

For any integer $\ell \geq \tau$, any $h \in \mathbb{L}^{2}([0,1])$ can be expanded on $\mathcal{B}$ as

$$
h(x)=\sum_{k=0}^{2^{\ell}-1} \alpha_{\ell, k} \phi_{\ell, k}(x)+\sum_{j=\ell}^{\infty} \sum_{k=0}^{2^{j}-1} \beta_{j, k} \psi_{j, k}(x), \quad x \in[0,1],
$$


where $\alpha_{j, k}$ and $\beta_{j, k}$ are the wavelet coefficients of $h$ defined by

$$
\alpha_{j, k}=\int_{0}^{1} h(x) \phi_{j, k}(x) d x, \quad \beta_{j, k}=\int_{0}^{1} h(x) \psi_{j, k}(x) d x .
$$

Besov balls. Let $M>0, s>0, p \geq 1$ and $r \geq 1$. A function $h$ belongs to $B_{p, r}^{s}(M)$ if and only if there exists a constant $M^{*}>0$ (depending on $M$ ) such that the associated wavelet coefficients (5) satisfy

$$
\begin{aligned}
2^{\tau(1 / 2-1 / p)}\left(\sum_{k=0}^{2^{\tau}-1}\left|\alpha_{\tau, k}\right|^{p}\right)^{1 / p} & +\left(\sum_{j=\tau}^{\infty}\left(2^{j(s+1 / 2-1 / p)}\left(\sum_{k=0}^{2^{j}-1}\left|\beta_{j, k}\right|^{p}\right)^{1 / p}\right)^{r}\right)^{1 / r} \\
\leq M^{*} &
\end{aligned}
$$

In this expression, $s$ is a smoothness parameter and $p$ and $r$ are norm parameters. For a particular choice of $s, p$ and $r, B_{p, r}^{s}(M)$ contain the Hölder and Sobolev balls. See Meyer (1992).

\section{Estimators}

Estimators of the wavelet coefficients. The first step to estimate $f_{\sigma^{2}}$ consists in expanding $f_{\sigma^{2}}$ on $\mathcal{B}$ and estimating its unknown wavelet coefficients. For any positive integer $\ell$ and any $h \in \mathcal{C}^{\ell}([0,1])$, set

$$
T(h)(x)=(x h(x))^{\prime}, \quad T_{\ell}(h)(x)=T\left(T_{\ell-1}(h)\right)(x), \quad x \in[0,1] .
$$

For any integer $j \geq \tau$ and any $k \in\left\{0, \ldots, 2^{j}-1\right\}$,

- we estimate $\alpha_{j, k}=\int_{0}^{1} f_{\sigma^{2}}(x) \phi_{j, k}(x) d x$ by

$$
\widehat{\alpha}_{j, k}=\frac{1}{n} \sum_{i=1}^{n} T_{\nu}\left(\phi_{j, k}\right)\left(S_{i}\right),
$$

( $\nu$ is the one in $(3))$

- we estimate $\beta_{j, k}=\int_{0}^{1} f_{\sigma^{2}}(x) \psi_{j, k}(x) d x$ by

$$
\widehat{\beta}_{j, k}=\frac{1}{n} \sum_{i=1}^{n} T_{\nu}\left(\psi_{j, k}\right)\left(S_{i}\right) .
$$

Some statistical properties of $\widehat{\alpha}_{j, k}$ and $\widehat{\beta}_{j, k}$ are studied in Propositions 2 and 3 .

We consider two wavelets estimators for $f_{\sigma^{2}}$ : a linear estimator and a hard thresholding estimator. 
Linear estimator. Assuming that $f_{\sigma^{2}} \in B_{p, r}^{s}(M)$ with $p \geq 2$, we define the linear estimator $\widehat{f}^{L}$ by

$$
\widehat{f}^{L}(x)=\sum_{k=0}^{2^{j_{0}}-1} \widehat{\alpha}_{j_{0}, k} \phi_{j_{0}, k}(x), \quad x \in[0,1],
$$

where $\widehat{\alpha}_{j, k}$ is defined by (7) and $j_{0}$ is an integer which will be chosen later (see Theorem 1 below).

For a survey on wavelet linear estimators for various density models, we refer to Chaubey et al. (2010a).

Hard thresholding estimator. We define the hard thresholding estimator $\widehat{f}^{H}$ by

$$
\widehat{f}^{H}(x)=\sum_{k=0}^{2^{\tau}-1} \widehat{\alpha}_{\tau, k} \phi_{\tau, k}(x)+\sum_{j=\tau}^{j_{1}} \sum_{k=0}^{2^{j}-1} \widehat{\beta}_{j, k} \mathbf{1}_{\left\{\left|\widehat{\beta}_{j, k}\right| \geq \kappa \lambda_{j}\right\}} \psi_{j, k}(x),
$$

$x \in[0,1]$, where $\widehat{\alpha}_{\tau, k}$ is defined by $(7), \widehat{\beta}_{j, k}$ by $(8), j_{1}$ is the integer satisfying

$$
\begin{gathered}
\frac{1}{2}\left(\frac{n_{*}}{\ln n_{*}}\right)^{1 /(2 \nu+1)}<2^{j_{1}} \leq\left(\frac{n_{*}}{\ln n_{*}}\right)^{1 /(2 \nu+1)}, \\
n_{*}=n^{\theta /(\theta+1)},
\end{gathered}
$$

$\theta$ is the one in (2), $\kappa$ is a large enough constant (the one in Proposition 3 ) and

$$
\lambda_{j}=2^{\nu j} \sqrt{\frac{\ln n_{*}}{n_{*}}} .
$$

The feature of the hard thresholding estimator is to only estimate the "large" unknown wavelet coefficients of $f_{\sigma^{2}}$ which contain the main characteristics of $f_{\sigma^{2}}$.

For the construction of hard thresholding wavelet estimators in the standard density model, see e.g. Donoho et al. (1996) and Delyon and Juditsky (1996), in the (standard) convolution density model, see e.g. Pensky and Vidakovic (1999) and Fan and Koo (2002), and in the density model under multiplicative censoring, see Chaubey et al. (2010b).

\section{Results}

Upper bounds for $\widehat{f}^{L}$ and $\widehat{f}^{H}$ are given in Theorems 1 and 2 below.

Theorem 1 Consider (1) under the assumptions of Section 2. Suppose that $f_{\sigma^{2}} \in B_{p, r}^{s}(M)$ with $s>0, p \geq 2$ and $r \geq 1$. For any $q \in(0,1)$, let $\widehat{f}^{L}$ be (9) with $j_{0}$ such that

$$
\frac{1}{2} n^{1 /(2 s+2 \nu+1+q)}<2^{j_{0}} \leq n^{1 /(2 s+2 \nu+1+q)} .
$$


Then there exists a constant $C>0$ such that

$$
\mathbb{E}\left(\int_{0}^{1}\left(\widehat{f}^{L}(x)-f_{\sigma^{2}}(x)\right)^{2} d x\right) \leq C n^{-2 s /(2 s+2 \nu+1+q)} .
$$

Theorem 2 Consider (1) under the assumptions of Section 2 . Let $\widehat{f}^{H}$ be (10). Suppose that $f_{\sigma^{2}} \in B_{p, r}^{s}(M)$ with $r \geq 1,\{p \geq 2$ and $s>0\}$ or $\{p \in[1,2)$ and $s>(2 \nu+1) / p\}$. Then there exists a constant $C>0$ such that

$$
\mathbb{E}\left(\int_{0}^{1}\left(\widehat{f}^{H}(x)-f_{\sigma^{2}}(x)\right)^{2} d x\right) \leq C\left(\frac{\ln n_{*}}{n_{*}}\right)^{2 s /(2 s+2 \nu+1)}
$$

where $n_{*}=n^{\theta /(\theta+1)}$ and $\theta$ is the one in (2).

The proof of Theorem 2 is based on several probability results (moment inequalities, concentration inequality,...) and a suitable decomposition of the MISE.

Theorem 2 shows that, besides being adaptive, $\widehat{f}^{H}$ attains a rate of convergence close to the one of $\widehat{f}^{L}$.

Note that, if we restrict our study to the independent case i.e. $\theta \rightarrow \infty$ and $\nu=1$, the rate of convergence attained by $\widehat{f}^{H}$ becomes the one for the classical density model under multiplicative censoring i.e. $(\ln n / n)^{2 s /(2 s+3)}$. See (Chaubey et al. 2010b, Theorem 5.2) with $\rho_{n}=1$.

Conclusion and perspectives. We construct a new adaptive estimator $\widehat{f}^{H}$ for $f_{\sigma^{2}}$ from (1). It is based on wavelets and thresholding. It attains a sharp rate of convergence over Besov balls.

A possible perspective of this work is to consider other realistic dependence conditions (polynomial strongly mixing dependence, associated sequences, ... ).

Moreover, perhaps we can improve the estimation of $f_{\sigma^{2}}$ by considering other kinds of thresholding rules as the block thresholding one (BlockJS, ... ). See e.g. Cai $(1999,2002)$ and Chesneau et al. (2010). However, to bound the MISE of such block thresholding estimators (as mentioned in (Van Zanten and Zareba $2008,3.3)$ ), it is not immediately clear how to extend some technical results (Talagrand's inequality, ... ) to the dependent case.

All these aspects need further investigations that we leave for a future work.

\section{Proofs}

In this section, we consider (1) under the assumptions of Section 2. Moreover, $C$ denotes any constant that does not depend on $j, k$ and $n$. Its value may change from one term to another and may depends on $\phi$ or $\psi$. 
6.1 Auxiliary results

Lemma 1 For any positive integer $\ell$ and any $h \in \mathcal{C}^{\ell}([0,1])$, set

$$
G(h)(x)=-x h^{\prime}(x), \quad G_{\ell}(h)(x)=G\left(G_{\ell-1}(h)\right)(x) .
$$

1. We have

$$
f_{\sigma^{2}}(x)=G_{\nu}\left(f_{S}\right)(x), \quad x \in[0,1],
$$

2. Let $T_{\nu}$ be (6). We have, for any $h \in \mathcal{C}^{\nu}([0,1])$,

$$
\int_{0}^{1} f_{\sigma^{2}}(x) h(x) d x=\int_{0}^{1} f_{S}(x) T_{\nu}(h)(x) d x .
$$

\section{Proof of Lemma 1.}

1. By definition of $f_{Z}$ and the Binomial theorem, we obtain

$$
\begin{aligned}
f_{S}(x) & =\int_{x}^{1} f_{Z}\left(\frac{x}{y}\right) f_{\sigma^{2}}(y) \frac{1}{y} d y \\
& =\frac{1}{(\nu-1) !} \int_{x}^{1}(\ln y-\ln x)^{\nu-1} f_{\sigma^{2}}(y) \frac{1}{y} d y \\
& =\frac{1}{(\nu-1) !} \sum_{u=0}^{\nu-1}\left(\begin{array}{c}
\nu-1 \\
u
\end{array}\right)(-\ln x)^{u} \int_{x}^{1}(\ln y)^{\nu-1-u} f_{\sigma^{2}}(y) \frac{1}{y} d y .
\end{aligned}
$$

Therefore, using $\sum_{u=0}^{\nu-1}\left(\begin{array}{c}\nu-1 \\ u\end{array}\right)(-1)^{u}=0$,

$$
\begin{aligned}
f_{S}^{\prime}(x) & =-\frac{1}{(\nu-1) !} \sum_{u=1}^{\nu-1}\left(\begin{array}{c}
\nu-1 \\
u
\end{array}\right) \frac{u}{x}(-\ln x)^{u-1} \int_{x}^{1}(\ln y)^{\nu-1-u} f_{\sigma^{2}}(y) \frac{1}{y} d y \\
& -\frac{1}{(\nu-1) !} \sum_{u=0}^{\nu-1}\left(\begin{array}{c}
\nu-1 \\
u
\end{array}\right)(-\ln x)^{u}(\ln x)^{\nu-1-u} f_{\sigma^{2}}(x) \frac{1}{x} \\
& =-\frac{1}{x}\left(\frac{1}{(\nu-1) !} \sum_{u=1}^{\nu-1}\left(\begin{array}{c}
\nu-1 \\
u
\end{array}\right) u(-\ln x)^{u-1} \int_{x}^{1}(\ln y)^{\nu-1-u} f_{\sigma^{2}}(y) \frac{1}{y} d y\right) \\
& -(\ln x)^{\nu-1} f_{\sigma^{2}}(x) \frac{1}{x} \frac{1}{(\nu-1) !} \sum_{u=0}^{\nu-1}\left(\begin{array}{c}
\nu-1 \\
u
\end{array}\right)(-1)^{u} \\
& =-\frac{1}{x}\left(\frac{1}{(\nu-1) !} \sum_{u=1}^{\nu-1}\left(\begin{array}{c}
\nu-1 \\
u
\end{array}\right) u(-\ln x)^{u-1} \int_{x}^{1}(\ln y)^{\nu-1-u} f_{\sigma^{2}}(y) \frac{1}{y} d y\right)
\end{aligned}
$$

So

$$
\begin{aligned}
G\left(f_{S}\right)(x) & =-x f_{S}^{\prime}(x) \\
& =\frac{1}{(\nu-1) !} \sum_{u=1}^{\nu-1}\left(\begin{array}{c}
\nu-1 \\
u
\end{array}\right) u(-\ln x)^{u-1} \int_{x}^{1}(\ln y)^{\nu-1-u} f_{\sigma^{2}}(y) \frac{1}{y} d y .
\end{aligned}
$$


For any $\ell \in\{1, \ldots, \nu-1\}$, proceeding $\ell$ times in a similar fashion to the above i.e. using derivations and the equality $\sum_{u=m}^{\nu-1}\left(\begin{array}{c}\nu-1 \\ u\end{array}\right) \frac{u !}{(u-m) !}(-1)^{u}=$ $\frac{(\nu-1) !}{(\nu-m-1) !}(-1)^{m} \sum_{u=0}^{\nu-m-1}\left(\begin{array}{c}\nu-m-1 \\ u\end{array}\right)(-1)^{u}=0, m \in\{0, \ldots, \ell\}$, we obtain

$$
G_{\ell}\left(f_{S}\right)(x)=\frac{1}{(\nu-1) !} \sum_{u=\ell}^{\nu-1}\left(\begin{array}{c}
\nu-1 \\
u
\end{array}\right) \frac{u !}{(u-\ell) !}(-\ln x)^{u-\ell} \int_{x}^{1}(\ln y)^{\nu-1-u} f_{\sigma^{2}}(y) \frac{1}{y} d y .
$$

Hence

$$
G_{\nu-1}\left(f_{S}\right)(x)=\int_{x}^{1} f_{\sigma^{2}}(y) \frac{1}{y} d y
$$

and, by a derivation,

$$
f_{\sigma^{2}}(x)=-x\left(G_{\nu-1}\left(f_{S}\right)(x)\right)^{\prime}=G_{\nu}\left(f_{S}\right)(x) .
$$

2. Using (12), an integration by parts and the fact that $G_{\nu-1}\left(f_{S}\right)(1)=0$, we obtain

$$
\begin{aligned}
\int_{0}^{1} f_{\sigma^{2}}(x) h(x) d x & =\int_{0}^{1} G_{\nu}\left(f_{S}\right)(x) h(x) d x \\
& =\int_{0}^{1}-x\left(G_{\nu-1}\left(f_{S}\right)(x)\right)^{\prime} h(x) d x \\
& =\left[-x G_{\nu-1}\left(f_{S}\right)(x) h(x)\right]_{0}^{1}+\int_{0}^{1} G_{\nu-1}\left(f_{S}\right)(x)(x h(x))^{\prime} d x \\
& =\int_{0}^{1} G_{\nu-1}\left(f_{S}\right)(x) T(h)(x) d x
\end{aligned}
$$

Proceeding $\nu-1$ times in a similar fashion to the above i.e. using $\nu-1$ integrations by parts, we have

$$
\begin{aligned}
\int_{0}^{1} G_{\nu-1}\left(f_{S}\right)(x) T(h)(x) d x & =\int_{0}^{1} G_{\nu-2}\left(f_{S}\right)(x) T_{2}(h)(x) d x=\ldots \\
& =\int_{0}^{1} f_{S}(x) T_{\nu}(h)(x) d x .
\end{aligned}
$$

Therefore

$$
\int_{0}^{1} f_{\sigma^{2}}(x) h(x) d x=\int_{0}^{1} f_{S}(x) T_{\nu}(h)(x) d x .
$$

This ends the proof of Lemma 1.

Proposition 1 Let $q \geq 2$, for any $j \geq \tau$ and any $k \in\left\{0, \ldots, 2^{j}-1\right\}, \alpha_{j, k}=$ $\int_{0}^{1} f_{\sigma^{2}}(x) \phi_{j, k}(x) d x$ and $T_{\nu}$ be $(6)$. Then

1.

$$
\mathbb{E}\left(T_{\nu}\left(\phi_{j, k}\right)\left(S_{1}\right)\right)=\alpha_{j, k}
$$


2.

$$
\sup _{x \in[0,1]}\left|T_{\nu}\left(\phi_{j, k}\right)(x)\right| \leq \theta_{1} 2^{(2 \nu+1) j / 2},
$$

3.

where $\theta_{1}=\nu ! \sum_{u=0}^{\nu} \sup _{x \in[1-N, N]}\left|\phi^{(u)}(x)\right|$.

$$
\mathbb{E}\left(\left(T_{\nu}\left(\phi_{j, k}\right)\left(S_{1}\right)\right)^{2}\right) \leq \theta_{2} 2^{2 \nu j},
$$

where $\theta_{2}=C_{*} \nu(\nu !)^{2} \sum_{u=0}^{\nu} \int_{1-N}^{N}\left(\phi^{(u)}(y)\right)^{2} d y$.

4. for any $q \in(0,1)$, there exists a constant $C>0$ such that

$$
\mathbb{V}\left(\sum_{i=1}^{n} T_{\nu}\left(\phi_{j, k}\right)\left(S_{i}\right)\right) \leq C n 2^{(2 \nu+q) j}
$$

These results hold for $\psi$ instead of $\phi$ (and, a fortiori, $\beta_{j, k}=\int_{0}^{1} f_{\sigma^{2}}(x) \psi_{j, k}(x) d x$ instead of $\alpha_{j, k}$ ).

\section{Proof of Proposition 1.}

1. Since $\phi \in \mathcal{C}^{\nu}([0,1])$, it follows from Lemma 1 that

$$
\mathbb{E}\left(T_{\nu}\left(\phi_{j, k}\right)\left(S_{1}\right)\right)=\int_{0}^{1} T_{\nu}\left(\phi_{j, k}\right)(x) f_{S}(x) d x=\int_{0}^{1} f_{\sigma^{2}}(x) \phi_{j, k}(x)=\alpha_{j, k}
$$

2. For any $u \in\{0, \ldots, \nu\}$, we have $\left(\phi_{j, k}\right)^{(u)}(x)=2^{(2 u+1) j / 2} \phi^{(u)}\left(2^{j} x-k\right)$. Hence

$$
\begin{aligned}
\sup _{x \in[0,1]}\left|T_{\nu}\left(\phi_{j, k}\right)(x)\right| & \leq \nu ! \sum_{u=0}^{\nu} \sup _{x \in[0,1]}\left|x^{u}\left(\phi_{j, k}\right)^{(u)}(x)\right| \\
& \leq \nu ! \sum_{u=0}^{\nu} 2^{(2 u+1) j / 2} \sup _{x \in[1-N, N]}\left|\phi^{(u)}(x)\right| \\
& \leq \theta_{1} 2^{(2 \nu+1) j / 2} .
\end{aligned}
$$

3. Since $S_{1}(\Omega)=[0,1]$, we have

$$
\begin{aligned}
\mathbb{E}\left(\left(T_{\nu}\left(\phi_{j, k}\right)\left(S_{1}\right)\right)^{2}\right) & \leq \nu(\nu !)^{2} \sum_{u=0}^{\nu} \mathbb{E}\left(S_{1}^{2 u}\left(\left(\phi_{j, k}\right)^{(u)}\left(S_{1}\right)\right)^{2}\right) \\
& \leq \nu(\nu !)^{2} \sum_{u=0}^{\nu} \mathbb{E}\left(\left(\left(\phi_{j, k}\right)^{(u)}\left(S_{1}\right)\right)^{2}\right) .
\end{aligned}
$$


Using $(4),\left(\phi_{j, k}\right)^{(u)}(x)=2^{(2 u+1) j / 2} \phi^{(u)}\left(2^{j} x-k\right)$ and doing the change of variables $y=2^{j} x-k$, we obtain

$$
\begin{aligned}
\mathbb{E}\left(\left(\left(\phi_{j, k}\right)^{(u)}\left(S_{1}\right)\right)^{2}\right) & =\int_{0}^{1}\left(\left(\phi_{j, k}\right)^{(u)}(x)\right)^{2} f_{S}(x) d x \leq C_{*} \int_{0}^{1}\left(\left(\phi_{j, k}\right)^{(u)}(x)\right)^{2} d x \\
& \leq C_{*} 2^{(2 u+1) j} \int_{0}^{1}\left(\phi^{(u)}\left(2^{j} x-k\right)\right)^{2} d x \\
& =C_{*} 2^{2 u j} \int_{-k}^{2^{j}-k}\left(\phi^{(u)}(y)\right)^{2} d y \\
& \leq C_{*} 2^{2 u j} \int_{1-N}^{N}\left(\phi^{(u)}(y)\right)^{2} d y
\end{aligned}
$$

Putting (15) and (16) together, we obtain

$$
\begin{aligned}
\mathbb{E}\left(\left(T_{\nu}\left(\phi_{j, k}\right)\left(S_{1}\right)\right)^{2}\right) & \leq C_{*} \nu(\nu !)^{2} \sum_{u=0}^{\nu} 2^{2 u j} \int_{1-N}^{N}\left(\phi^{(u)}(y)\right)^{2} d y \\
& \leq \theta_{2} 2^{2 \nu j}
\end{aligned}
$$

4. We have

$$
\begin{aligned}
& \mathbb{V}\left(\sum_{i=1}^{n} T_{\nu}\left(\phi_{j, k}\right)\left(S_{i}\right)\right) \\
& =n \mathbb{V}\left(T_{\nu}\left(\phi_{j, k}\right)\left(S_{1}\right)\right)+2 \sum_{v=2}^{n} \sum_{\ell=1}^{v-1} \mathbb{C}\left(T_{\nu}\left(\phi_{j, k}\right)\left(S_{v}\right), T_{\nu}\left(\phi_{j, k}\right)\left(S_{\ell}\right)\right) \\
& \leq n \mathbb{V}\left(T_{\nu}\left(\phi_{j, k}\right)\left(S_{1}\right)\right)+2\left|\sum_{v=2}^{n} \sum_{\ell=1}^{v-1} \mathbb{C}\left(T_{\nu}\left(\phi_{j, k}\right)\left(S_{v}\right), T_{\nu}\left(\phi_{j, k}\right)\left(S_{\ell}\right)\right)\right| .
\end{aligned}
$$

Using (17), we have

$$
\mathbb{V}\left(T_{\nu}\left(\phi_{j, k}\right)\left(S_{1}\right)\right) \leq \mathbb{E}\left(\left(T_{\nu}\left(\phi_{j, k}\right)\left(S_{1}\right)\right)^{2}\right) \leq C 2^{2 \nu j}
$$

The stationarity of $\left(S_{i}\right)_{i \in \mathbb{Z}}$ implies that

$$
\begin{aligned}
& \left|\sum_{v=2}^{n} \sum_{\ell=1}^{v-1} \mathbb{C}\left(T_{\nu}\left(\phi_{j, k}\right)\left(S_{v}\right), T_{\nu}\left(\phi_{j, k}\right)\left(S_{\ell}\right)\right)\right| \\
& =\left|\sum_{m=1}^{n}(n-m) \mathbb{C}\left(T_{\nu}\left(\phi_{j, k}\right)\left(S_{0}\right), T_{\nu}\left(\phi_{j, k}\right)\left(S_{m}\right)\right)\right| \\
& \leq n \sum_{m=1}^{n}\left|\mathbb{C}\left(T_{\nu}\left(\phi_{j, k}\right)\left(S_{0}\right), T_{\nu}\left(\phi_{j, k}\right)\left(S_{m}\right)\right)\right| .
\end{aligned}
$$


By the Davydov inequality (see Davydov (1970)), it holds that

$$
\begin{aligned}
& \left|\mathbb{C}\left(T_{\nu}\left(\phi_{j, k}\right)\left(S_{0}\right), T_{\nu}\left(\phi_{j, k}\right)\left(S_{m}\right)\right)\right| \\
& \leq 10 a_{m}^{q}\left(\mathbb{E}\left(\left(T_{\nu}\left(\phi_{j, k}\right)\left(S_{0}\right)\right)^{2 /(1-q)}\right)\right)^{1-q} \\
& \leq 10 a_{m}^{q}\left(\sup _{x \in[0,1]}\left|T_{\nu}\left(\phi_{j, k}\right)(x)\right|\right)^{2 q}\left(\mathbb{E}\left(\left(T_{\nu}\left(\phi_{j, k}\right)\left(S_{0}\right)\right)^{2}\right)\right)^{1-q} .
\end{aligned}
$$

Using (14) and (17) we obtain

$$
\begin{aligned}
\left|\mathbb{C}\left(T_{\nu}\left(\phi_{j, k}\right)\left(S_{0}\right), T_{\nu}\left(\phi_{j, k}\right)\left(S_{m}\right)\right)\right| & \leq C a_{m}^{q}\left(2^{(2 \nu+1) j / 2}\right)^{2 q}\left(2^{2 \nu j}\right)^{1-q} \\
& \leq C 2^{(2 \nu+q) j} a_{m}^{q} .
\end{aligned}
$$

Since $\sum_{m=1}^{n} a_{m}^{q} \leq \sum_{m=1}^{\infty} a_{m}^{q}=C$, we have

$$
\begin{aligned}
\left|\sum_{v=2}^{n} \sum_{\ell=1}^{v-1} \mathbb{C}\left(T_{\nu}\left(\phi_{j, k}\right)\left(S_{v}\right), T_{\nu}\left(\phi_{j, k}\right)\left(S_{\ell}\right)\right)\right| & \leq C n 2^{(2 \nu+q) j} \sum_{m=1}^{n} a_{m}^{q} \\
& \leq C n 2^{(2 \nu+q) j} .
\end{aligned}
$$

It follows from (18), (19) and (20) that

$$
\mathbb{V}\left(\sum_{i=1}^{n} T_{\nu}\left(\phi_{j, k}\right)\left(S_{i}\right)\right) \leq C n 2^{(2 \nu+q) j} .
$$

The proof of Proposition 1 is complete.

Proposition 2 For any integer $j \geq \tau$ and any $k \in\left\{0, \ldots, 2^{j}-1\right\}$, let $\alpha_{j, k}=$ $\int_{0}^{1} f_{\sigma^{2}}(x) \phi_{j, k}(x) d x$ and $\widehat{\alpha}_{j, k}$ be $(7)$. Then, for any $q \in(0,1)$,

1. there exists a constant $C>0$ such that

$$
\mathbb{E}\left(\left(\widehat{\alpha}_{j, k}-\alpha_{j, k}\right)^{2}\right) \leq C 2^{(2 \nu+q) j} \frac{1}{n}
$$

2. there exists a constant $C>0$ such that

$$
\mathbb{E}\left(\left(\widehat{\alpha}_{j, k}-\alpha_{j, k}\right)^{4}\right) \leq C 2^{(4 \nu+1+q) j} \frac{1}{n} .
$$

These inequalities hold for $\widehat{\beta}_{j, k}$ defined by (8) instead of $\widehat{\alpha}_{j, k}$, and $\beta_{j, k}=$ $\int_{0}^{1} f_{\sigma^{2}}(x) \psi_{j, k}(x) d x$ instead of $\alpha_{j, k}$.

\section{Proof of Proposition 2.}


1. Using Proposition 1, we have

$$
\begin{aligned}
\mathbb{E}\left(\left(\widehat{\alpha}_{j, k}-\alpha_{j, k}\right)^{2}\right) & =\mathbb{E}\left(\left(\widehat{\alpha}_{j, k}-\mathbb{E}\left(\widehat{\alpha}_{j, k}\right)\right)^{2}\right)=\mathbb{V}\left(\widehat{\alpha}_{j, k}\right) \\
& =\frac{1}{n^{2}} \mathbb{V}\left(\sum_{i=1}^{n} T_{\nu}\left(\phi_{j, k}\right)\left(S_{i}\right)\right) \\
& \leq C \frac{1}{n^{2}} n 2^{(2 \nu+q) j}=C 2^{(2 \nu+q) j} \frac{1}{n} .
\end{aligned}
$$

2. The triangular inequality yields

$$
\left|\widehat{\alpha}_{j, k}-\alpha_{j, k}\right| \leq\left|\widehat{\alpha}_{j, k}\right|+\left|\alpha_{j, k}\right|
$$

Using again Proposition 1, we obtain

$$
\left|\widehat{\alpha}_{j, k}\right| \leq \frac{1}{n} \sum_{i=1}^{n}\left|T_{\nu}\left(\phi_{j, k}\right)\left(S_{i}\right)\right| \leq \sup _{x \in[0,1]}\left|T_{\nu}\left(\phi_{j, k}\right)(x)\right| \leq C 2^{(2 \nu+1) j / 2} .
$$

We have $\sup _{x \in[0,1]}\left|\phi_{j, k}(x)\right| \leq 2^{j / 2} \sup _{x \in[1-N, N]}|\phi(x)|=C 2^{j / 2}$. So

$$
\begin{aligned}
\left|\alpha_{j, k}\right| & \leq \int_{0}^{1} f_{\sigma^{2}}(x)\left|\phi_{j, k}(x)\right| d x \leq C 2^{j / 2} \int_{0}^{1} f_{\sigma^{2}}(x) d x=C 2^{j / 2} \\
& \leq C 2^{(2 \nu+1) j / 2} .
\end{aligned}
$$

Therefore

$$
\left|\widehat{\alpha}_{j, k}-\alpha_{j, k}\right| \leq C 2^{(2 \nu+1) j / 2} .
$$

It follows from (23) and (21) that

$$
\mathbb{E}\left(\left(\widehat{\alpha}_{j, k}-\alpha_{j, k}\right)^{4}\right) \leq C 2^{(2 \nu+1) j} \mathbb{E}\left(\left(\widehat{\alpha}_{j, k}-\alpha_{j, k}\right)^{2}\right) \leq C 2^{(4 \nu+1+q) j} \frac{1}{n} .
$$

The proof of Proposition 2 is complete.

Proposition 3 For any $j \in\left\{\tau, \ldots, j_{1}\right\}$ and any $k \in\left\{0, \ldots, 2^{j}-1\right\}$, let $\beta_{j, k}=$ $\int_{0}^{1} f_{\sigma^{2}}(x) \psi_{j, k}(x) d x, \widehat{\beta}_{j, k}$ be (8) and $\lambda_{j}$ be (11). Then there exist two constants, $\kappa>0$ and $C>0$, such that

$$
\mathbb{P}\left(\left|\widehat{\beta}_{j, k}-\beta_{j, k}\right| \geq \kappa \lambda_{j} / 2\right) \leq C \frac{1}{n_{*}^{4}}
$$

where $n_{*}=n^{\theta /(\theta+1)}$.

Proof of Proposition 3. Lemma 2 below presents a Bernstein inequality for exponentially strongly mixing process. This is a slightly modified version of (Modha and Masry 1996, Theorem 4.2). 
Lemma 2 (Modha and Masry (1996)) Let $\gamma>0, c>0, \theta>1$ and $\left(Z_{i}\right)_{i \in \mathbb{Z}}$ be a stationary process such that, for any $m \in \mathbb{Z}$, the associated $m$-th strongly mixing coefficient satisfies

$$
a_{m} \leq \gamma \exp \left(-c|m|^{\theta}\right) .
$$

Let $n \in \mathbb{N}^{*}, h: \mathbb{R} \rightarrow \mathbb{R}$ be a measurable function and, for any $i \in \mathbb{Z}, U_{i}=$ $h\left(Z_{i}\right)$. We assume that there exists a constant $M>0$ satisfying $\left|U_{1}\right| \leq M<$ $\infty$. Then, for any $\lambda>0$, we have

$$
\mathbb{P}\left(\left|\frac{1}{n} \sum_{i=1}^{n} U_{i}\right| \geq \lambda\right) \leq 2\left(1+4 e^{-2} \gamma\right) \exp \left(-\frac{u \lambda^{2} n^{\theta /(\theta+1)}}{2\left(\mathbb{E}\left(U_{1}^{2}\right)+\lambda M / 3\right)}\right),
$$

where $u=(1 / 2)(c / 8)^{1 /(\theta+1)}$.

For any $i \in\{1, \ldots, n\}$, set

$$
U_{i}=T_{\nu}\left(\psi_{j, k}\right)\left(S_{i}\right)-\beta_{j, k}
$$

Proposition 1 applied with $\psi$ instead of $\phi$ implies that

$$
\mathbb{E}\left(U_{1}\right)=0, \quad \mathbb{E}\left(U_{1}^{2}\right) \leq \mathbb{E}\left(\left(T_{\nu}\left(\psi_{j, k}\right)\left(S_{1}\right)\right)^{2}\right) \leq \theta_{2} 2^{2 \nu j}
$$

and, using (22),

$$
\left|U_{1}\right| \leq \sup _{x \in[0,1]}\left|T_{\nu}\left(\psi_{j, k}\right)(x)\right|+\left|\beta_{j, k}\right| \leq\left(\theta_{1}+\sup _{x \in[1-N, N]}|\psi(x)|\right) 2^{(2 \nu+1) j / 2} .
$$

It follows from Lemma 2 applied with $U_{1}, \ldots, U_{n}, \lambda=\kappa \lambda_{j} / 2$ and $M=\left(\theta_{1}+\right.$ $\left.\sup _{x \in[1-N, N]}|\psi(x)|\right) 2^{(2 \nu+1) j / 2}$, and the inequality $2^{j_{1}} \leq n_{*} / \ln n_{*}$ that

$$
\begin{aligned}
& \mathbb{P}\left(\left|\widehat{\beta}_{j, k}-\beta_{j, k}\right| \geq \kappa \lambda_{j} / 2\right)=\mathbb{P}\left(\left|\frac{1}{n} \sum_{i=1}^{n} U_{i}\right| \geq \kappa \lambda_{j} / 2\right) \\
& \leq C \exp \left(-\frac{u \kappa^{2} \lambda_{j}^{2} n_{*}}{8\left(\theta_{2} 2^{2 \nu j}+\kappa\left(\theta_{1}+\sup _{x \in[1-N, N]}|\psi(x)|\right) \lambda_{j} 2^{(2 \nu+1) j / 2} / 6\right)}\right) \\
& \leq C \exp \left(-\frac{u \kappa^{2} \ln n_{*}}{8\left(\theta_{2}+\kappa\left(\theta_{1}+\sup _{x \in[1-N, N]}|\psi(x)|\right) 2^{j / 2} \sqrt{\ln n_{*} / n_{*}} / 6\right)}\right) \\
& \leq C \frac{1}{n_{*}^{\rho(\kappa)}},
\end{aligned}
$$

where

$$
\rho(\kappa)=\frac{u \kappa^{2}}{8\left(\theta_{2}+\kappa\left(\theta_{1}+\sup _{x \in[1-N, N]}|\psi(x)|\right) / 6\right)} .
$$

Since $\lim _{x \rightarrow \infty} \rho(x)=\infty$, there exists a $\kappa>0$ such that $\rho(\kappa)=4$. Therefore, for such a $\kappa$, we have

$$
\mathbb{P}\left(\left|\widehat{\beta}_{j, k}-\beta_{j, k}\right| \geq \kappa \lambda_{j} / 2\right) \leq C \frac{1}{n_{*}^{4}} .
$$

This completes the proof of Proposition 3. 
6.2 Proofs of the main results

Proof of Theorem 1. We expand the function $f_{\sigma^{2}}$ on $\mathcal{B}$ as

$$
f_{\sigma^{2}}(x)=\sum_{k=0}^{2^{j} 0-1} \alpha_{j_{0}, k} \phi_{j_{0}, k}(x)+\sum_{j=j_{0}}^{\infty} \sum_{k=0}^{2^{j}-1} \beta_{j, k} \psi_{j, k}(x), \quad x \in[0,1],
$$

where

$$
\alpha_{j_{0}, k}=\int_{0}^{1} f_{\sigma^{2}}(x) \phi_{j_{0}, k}(x) d x, \quad \beta_{j, k}=\int_{0}^{1} f_{\sigma^{2}}(x) \psi_{j, k}(x) d x .
$$

We have, for any $x \in[0,1]$,

$$
\widehat{f}^{L}(x)-f_{\sigma^{2}}(x)=\sum_{k=0}^{2^{j_{0}}-1}\left(\widehat{\alpha}_{j_{0}, k}-\alpha_{j_{0}, k}\right) \phi_{j_{0}, k}(x)-\sum_{j=j_{0}}^{\infty} \sum_{k=0}^{2^{j}-1} \beta_{j, k} \psi_{j, k}(x) .
$$

Since $\mathcal{B}$ is an orthonormal basis of $\mathbb{L}^{2}([0,1])$, we have

$$
\mathbb{E}\left(\int_{0}^{1}\left(\widehat{f}^{L}(x)-f_{\sigma^{2}}(x)\right)^{2} d x\right)=\sum_{k=0}^{2^{j} 0-1} \mathbb{E}\left(\left(\widehat{\alpha}_{j_{0}, k}-\alpha_{j_{0}, k}\right)^{2}\right)+\sum_{j=j_{0}}^{\infty} \sum_{k=0}^{2^{j}-1} \beta_{j, k}^{2}
$$

Using Proposition 2, we obtain

$$
\sum_{k=0}^{2^{j_{0}}-1} \mathbb{E}\left(\left(\widehat{\alpha}_{j_{0}, k}-\alpha_{j_{0}, k}\right)^{2}\right) \leq C 2^{j_{0}(2 \nu+1+q)} \frac{1}{n} \leq C n^{-2 s /(2 s+2 \nu+1+q)}
$$

Since $p \geq 2$, we have $B_{p, r}^{s}(M) \subseteq B_{2, \infty}^{s}(M)$. Hence

$$
\sum_{j=j_{0}}^{\infty} \sum_{k=0}^{2^{j}-1} \beta_{j, k}^{2} \leq C 2^{-2 j_{0} s} \leq C n^{-2 s /(2 s+2 \nu+1+q)}
$$

Therefore

$$
\mathbb{E}\left(\int_{0}^{1}\left(\widehat{f}^{L}(x)-f_{\sigma^{2}}(x)\right)^{2} d x\right) \leq C n^{-2 s /(2 s+2 \nu+1+q)} .
$$

The proof of Theorem 1 is complete. 
Proof of Theorem 2. We expand the function $f_{\sigma^{2}}$ on $\mathcal{B}$ as

$$
f_{\sigma^{2}}(x)=\sum_{k=0}^{2^{\tau}-1} \alpha_{\tau, k} \phi_{\tau, k}(x)+\sum_{j=\tau}^{\infty} \sum_{k=0}^{2^{j}-1} \beta_{j, k} \psi_{j, k}(x), \quad x \in[0,1],
$$

where

$$
\alpha_{\tau, k}=\int_{0}^{1} f_{\sigma^{2}}(x) \phi_{\tau, k}(x) d x, \quad \beta_{j, k}=\int_{0}^{1} f_{\sigma^{2}}(x) \psi_{j, k}(x) d x .
$$

We have, for any $x \in[0,1]$,

$$
\begin{aligned}
& \widehat{f}^{H}(x)-f_{\sigma^{2}}(x) \\
& =\sum_{k=0}^{2^{\tau}-1}\left(\widehat{\alpha}_{\tau, k}-\alpha_{\tau, k}\right) \phi_{\tau, k}(x)+\sum_{j=\tau}^{j_{1}} \sum_{k=0}^{2^{j}-1}\left(\widehat{\beta}_{j, k} \mathbf{1}_{\left\{\left|\widehat{\beta}_{j, k}\right| \geq \kappa \lambda_{j}\right\}}-\beta_{j, k}\right) \psi_{j, k}(x) \\
& -\sum_{j=j_{1}+1}^{\infty} \sum_{k=0}^{2^{j}-1} \beta_{j, k} \psi_{j, k}(x) .
\end{aligned}
$$

Since $\mathcal{B}$ is an orthonormal basis of $\mathbb{L}^{2}([0,1])$, we have

$$
\mathbb{E}\left(\int_{0}^{1}\left(\widehat{f}^{H}(x)-f_{\sigma^{2}}(x)\right)^{2} d x\right)=R+S+T
$$

where

$R=\sum_{k=0}^{2^{\tau}-1} \mathbb{E}\left(\left(\widehat{\alpha}_{\tau, k}-\alpha_{\tau, k}\right)^{2}\right), \quad S=\sum_{j=\tau}^{j_{1}} \sum_{k=0}^{2^{j}-1} \mathbb{E}\left(\left(\widehat{\beta}_{j, k} \mathbf{1}_{\left\{\left|\widehat{\beta}_{j, k}\right| \geq \kappa \lambda_{j}\right\}}-\beta_{j, k}\right)^{2}\right)$

and

$$
T=\sum_{j=j_{1}+1}^{\infty} \sum_{k=0}^{2^{j}-1} \beta_{j, k}^{2}
$$

Let us bound $R, T$ and $S$, in turn.

Using Proposition $2, n_{*}<n$ and $2 s /(2 s+2 \nu+1)<1$, we obtain

$$
R \leq C 2^{\tau(2 \nu+1+q)} \frac{1}{n} \leq C \frac{1}{n} \leq C \frac{1}{n_{*}} \leq C\left(\frac{\ln n_{*}}{n_{*}}\right)^{2 s /(2 s+2 \nu+1)} .
$$

For $r \geq 1$ and $p \geq 2$, we have $B_{p, r}^{s}(M) \subseteq B_{2, \infty}^{s}(M)$. Since $2 s /(2 s+2 \nu+1)<$ $2 s /(2 \nu+1)$, we have

$$
T \leq C \sum_{j=j_{1}+1}^{\infty} 2^{-2 j s} \leq C 2^{-2 j_{1} s} \leq C\left(\frac{\ln n_{*}}{n_{*}}\right)^{2 s /(2 \nu+1)} \leq C\left(\frac{\ln n_{*}}{n_{*}}\right)^{2 s /(2 s+2 \nu+1)} .
$$


For $r \geq 1$ and $p \in[1,2)$, we have $B_{p, r}^{s}(M) \subseteq B_{2, \infty}^{s+1 / 2-1 / p}(M)$. Since $s>$ $(2 \nu+1) / p$, we have $(s+1 / 2-1 / p) /(2 \nu+1)>s /(2 s+2 \nu+1)$. So

$$
\begin{aligned}
T & \leq C \sum_{j=j_{1}+1}^{\infty} 2^{-2 j(s+1 / 2-1 / p)} \leq C 2^{-2 j_{1}(s+1 / 2-1 / p)} \\
& \leq C\left(\frac{\ln n_{*}}{n_{*}}\right)^{2(s+1 / 2-1 / p) /(2 \nu+1)} \leq C\left(\frac{\ln n_{*}}{n_{*}}\right)^{2 s /(2 s+2 \nu+1)} .
\end{aligned}
$$

Hence, for $r \geq 1,\{p \geq 2$ and $s>0\}$ or $\{p \in[1,2)$ and $s>(2 \nu+1) / p\}$, we have

$$
T \leq C\left(\frac{\ln n_{*}}{n_{*}}\right)^{2 s /(2 s+2 \nu+1)} .
$$

We can write the term $S$ as

$$
S=S_{1}+S_{2}+S_{3}+S_{4}
$$

where

$$
\begin{gathered}
S_{1}=\sum_{j=\tau}^{j_{1}} \sum_{k=0}^{2^{j}-1} \mathbb{E}\left(\left(\widehat{\beta}_{j, k}-\beta_{j, k}\right)^{2} \mathbf{1}_{\left\{\left|\widehat{\beta}_{j, k}\right| \geq \kappa \lambda_{j}\right\}} \mathbf{1}_{\left\{\left|\beta_{j, k}\right|<\kappa \lambda_{j} / 2\right\}}\right), \\
S_{2}=\sum_{j=\tau}^{j_{1}} \sum_{k=0}^{2^{j}-1} \mathbb{E}\left(\left(\widehat{\beta}_{j, k}-\beta_{j, k}\right)^{2} \mathbf{1}_{\left\{\left|\widehat{\beta}_{j, k}\right| \geq \kappa \lambda_{j}\right\}} \mathbf{1}_{\left\{\left|\beta_{j, k}\right| \geq \kappa \lambda_{j} / 2\right\}}\right), \\
S_{3}=\sum_{j=\tau}^{j_{1}} \sum_{k=0}^{2^{j}-1} \mathbb{E}\left(\beta_{j, k}^{2} \mathbf{1}_{\left\{\left|\widehat{\beta}_{j, k}\right|<\kappa \lambda_{j}\right\}} \mathbf{1}_{\left\{\left|\beta_{j, k}\right| \geq 2 \kappa \lambda_{j}\right\}}\right)
\end{gathered}
$$

and

$$
S_{4}=\sum_{j=\tau}^{j_{1}} \sum_{k=0}^{2^{j}-1} \mathbb{E}\left(\beta_{j, k}^{2} \mathbf{1}_{\left\{\left|\widehat{\beta}_{j, k}\right|<\kappa \lambda_{j}\right\}} \mathbf{1}_{\left\{\left|\beta_{j, k}\right|<2 \kappa \lambda_{j}\right\}}\right) .
$$

Let us investigate the bounds of $S_{1}, S_{2}, S_{3}$ and $S_{4}$ in turn.

Upper bounds for $S_{1}$ and $S_{3}$. We have

$$
\begin{aligned}
& \left\{\left|\widehat{\beta}_{j, k}\right|<\kappa \lambda_{j},\left|\beta_{j, k}\right| \geq 2 \kappa \lambda_{j}\right\} \subseteq\left\{\left|\widehat{\beta}_{j, k}-\beta_{j, k}\right|>\kappa \lambda_{j} / 2\right\}, \\
& \left\{\left|\widehat{\beta}_{j, k}\right| \geq \kappa \lambda_{j},\left|\beta_{j, k}\right|<\kappa \lambda_{j} / 2\right\} \subseteq\left\{\left|\widehat{\beta}_{j, k}-\beta_{j, k}\right|>\kappa \lambda_{j} / 2\right\}
\end{aligned}
$$

and

$$
\left\{\left|\widehat{\beta}_{j, k}\right|<\kappa \lambda_{j},\left|\beta_{j, k}\right| \geq 2 \kappa \lambda_{j}\right\} \subseteq\left\{\left|\beta_{j, k}\right| \leq 2\left|\widehat{\beta}_{j, k}-\beta_{j, k}\right|\right\}
$$

So

$$
\max \left(S_{1}, S_{3}\right) \leq C \sum_{j=\tau}^{j_{1}} \sum_{k=0}^{2^{j}-1} \mathbb{E}\left(\left(\widehat{\beta}_{j, k}-\beta_{j, k}\right)^{2} \mathbf{1}_{\left\{\left|\widehat{\beta}_{j, k}-\beta_{j, k}\right|>\kappa \lambda_{j} / 2\right\}}\right)
$$


It follows from the Cauchy-Schwarz inequality, Proposition 2 with $q \in(0, \max (1,(2 \nu+$ 1) $/ \theta)$ which implies $2^{q j} \leq 2^{q j_{1}} \leq n_{*}^{q /(2 \nu+1)} \leq n / n_{*}, 2^{j} \leq 2^{j_{1}} \leq n_{*}$ and Proposition 3 that

$$
\begin{aligned}
& \mathbb{E}\left(\left(\widehat{\beta}_{j, k}-\beta_{j, k}\right)^{2} \mathbf{1}_{\left\{\left|\widehat{\beta}_{j, k}-\beta_{j, k}\right|>\kappa \lambda_{j} / 2\right\}}\right) \\
& \leq\left(\mathbb{E}\left(\left(\widehat{\beta}_{j, k}-\beta_{j, k}\right)^{4}\right)\right)^{1 / 2}\left(\mathbb{P}\left(\left|\widehat{\beta}_{j, k}-\beta_{j, k}\right|>\kappa \lambda_{j} / 2\right)\right)^{1 / 2} \\
& \leq C\left(2^{(4 \nu+1+q) j} \frac{1}{n}\right)^{1 / 2}\left(\frac{1}{n_{*}^{4}}\right)^{1 / 2}=C 2^{2 \nu j} \frac{1}{n_{*}^{2}}\left(2^{(1+q) j} \frac{1}{n}\right)^{1 / 2} \leq C 2^{2 \nu j} \frac{1}{n_{*}^{2}} .
\end{aligned}
$$

Since $2 s /(2 s+2 \nu+1)<1$, we have

$$
\begin{aligned}
\max \left(S_{1}, S_{3}\right) & \leq C \frac{1}{n_{*}^{2}} \sum_{j=\tau}^{j_{1}} 2^{j(1+2 \nu)} \leq C \frac{1}{n_{*}^{2}} 2^{j_{1}(1+2 \nu)} \leq C \frac{1}{n_{*}} \\
& \leq C \frac{\ln n_{*}}{n_{*}} \leq C\left(\frac{\ln n_{*}}{n_{*}}\right)^{2 s /(2 s+2 \nu+1)} .
\end{aligned}
$$

Upper bound for $S_{2}$. Using again Proposition 2 with $q \in(0, \max (1,(2 \nu+1) / \theta)$ which implies $2^{q j} \leq 2^{q j_{1}} \leq n_{*}^{q /(2 \nu+1)} \leq n / n_{*}$, we obtain

$$
\begin{aligned}
\mathbb{E}\left(\left(\widehat{\beta}_{j, k}-\beta_{j, k}\right)^{2}\right) & \leq C 2^{(2 \nu+q) j} \frac{1}{n} \leq C 2^{2 \nu j}\left(2^{q j} \frac{1}{n}\right) \leq C 2^{2 \nu j} \frac{1}{n_{*}} \\
& \leq C 2^{2 \nu j} \frac{\ln n_{*}}{n_{*}}
\end{aligned}
$$

Hence

$$
S_{2} \leq C \frac{\ln n_{*}}{n_{*}} \sum_{j=\tau}^{j_{1}} 2^{2 \nu j} \sum_{k=0}^{2^{j}-1} \mathbf{1}_{\left\{\left|\beta_{j, k}\right|>\kappa \lambda_{j} / 2\right\}} .
$$

Let $j_{2}$ be the integer defined by

$$
\frac{1}{2}\left(\frac{n_{*}}{\ln n_{*}}\right)^{1 /(2 s+2 \nu+1)}<2^{j_{2}} \leq\left(\frac{n_{*}}{\ln n_{*}}\right)^{1 /(2 s+2 \nu+1)} .
$$

We have

$$
S_{2} \leq S_{2,1}+S_{2,2}
$$

where

$$
S_{2,1}=C \frac{\ln n_{*}}{n_{*}} \sum_{j=\tau}^{j_{2}} 2^{2 \nu j} \sum_{k=0}^{2^{j}-1} \mathbf{1}_{\left\{\left|\beta_{j, k}\right|>\kappa \lambda_{j} / 2\right\}}
$$

and

$$
S_{2,2}=C \frac{\ln n_{*}}{n_{*}} \sum_{j=j_{2}+1}^{j_{1}} 2^{2 \nu j} \sum_{k=0}^{2^{j}-1} \mathbf{1}_{\left\{\left|\beta_{j, k}\right|>\kappa \lambda_{j} / 2\right\}}
$$


We have

$$
S_{2,1} \leq C \frac{\ln n_{*}}{n_{*}} \sum_{j=\tau}^{j_{2}} 2^{j(1+2 \nu)} \leq C \frac{\ln n_{*}}{n_{*}} 2^{j_{2}(1+2 \nu)} \leq C\left(\frac{\ln n_{*}}{n_{*}}\right)^{2 s /(2 s+2 \nu+1)} .
$$

For $r \geq 1$ and $p \geq 2$, since $B_{p, r}^{s}(M) \subseteq B_{2, \infty}^{s}(M)$,

$$
\begin{aligned}
S_{2,2} & \leq C \frac{\ln n_{*}}{n_{*}} \sum_{j=j_{2}+1}^{j_{1}} 2^{2 \nu j} \frac{1}{\lambda_{j}^{2}} \sum_{k=0}^{2^{j}-1} \beta_{j, k}^{2} \leq C \sum_{j=j_{2}+1}^{\infty} \sum_{k=0}^{2^{j}-1} \beta_{j, k}^{2} \leq C 2^{-2 j_{2} s} \\
& \leq C\left(\frac{\ln n_{*}}{n_{*}}\right)^{2 s /(2 s+2 \nu+1)} .
\end{aligned}
$$

For $r \geq 1, p \in[1,2)$ and $s>(2 \nu+1) / p$, using $\mathbf{1}_{\left\{\left|\beta_{j, k}\right|>\kappa \lambda_{j} / 2\right\}} \leq C\left|\beta_{j, k}\right|^{p} / \lambda_{j}^{p}$, $B_{p, r}^{s}(M) \subseteq B_{2, \infty}^{s+1 / 2-1 / p}(M)$ and $(2 s+2 \nu+1)(2-p) / 2+(s+1 / 2-1 / p+\nu-$ $2 \nu / p) p=2 s$, we have

$$
\begin{aligned}
S_{2,2} & \leq C \frac{\ln n_{*}}{n_{*}} \sum_{j=j_{2}+1}^{j_{1}} 2^{2 \nu j} \frac{1}{\lambda_{j}^{p}} \sum_{k=0}^{2^{j}-1}\left|\beta_{j, k}\right|^{p} \\
& \leq C\left(\frac{\ln n_{*}}{n_{*}}\right)^{(2-p) / 2} \sum_{j=j_{2}+1}^{\infty} 2^{j \nu(2-p)} 2^{-j(s+1 / 2-1 / p) p} \\
& \leq C\left(\frac{\ln n_{*}}{n_{*}}\right)^{(2-p) / 2} 2^{-j_{2}(s+1 / 2-1 / p+\nu-2 \nu / p) p} \\
& \leq C\left(\frac{\ln n_{*}}{n_{*}}\right)^{2 s /(2 s+2 \nu+1)}
\end{aligned}
$$

So, for $r \geq 1,\{p \geq 2$ and $s>0\}$ or $\{p \in[1,2)$ and $s>(2 \nu+1) / p\}$, we have

$$
S_{2} \leq C\left(\frac{\ln n_{*}}{n_{*}}\right)^{2 s /(2 s+2 \nu+1)}
$$

Upper bound for $S_{4}$. We have

$$
S_{4} \leq \sum_{j=\tau}^{j_{1}} \sum_{k=0}^{2^{j}-1} \beta_{j, k}^{2} \mathbf{1}_{\left\{\left|\beta_{j, k}\right|<2 \kappa \lambda_{j}\right\}}
$$

Let $j_{2}$ be the integer (30). Then

$$
S_{4} \leq S_{4,1}+S_{4,2},
$$

where

$$
S_{4,1}=\sum_{j=\tau}^{j_{2}} \sum_{k=0}^{2^{j}-1} \beta_{j, k}^{2} \mathbf{1}_{\left\{\left|\beta_{j, k}\right|<2 \kappa \lambda_{j}\right\}}, \quad S_{4,2}=\sum_{j=j_{2}+1}^{j_{1}} \sum_{k=0}^{2^{j}-1} \beta_{j, k}^{2} \mathbf{1}_{\left\{\left|\beta_{j, k}\right|<2 \kappa \lambda_{j}\right\}} .
$$


We have

$$
\begin{aligned}
S_{4,1} & \leq C \sum_{j=\tau}^{j_{2}} 2^{j} \lambda_{j}^{2}=C \frac{\ln n_{*}}{n_{*}} \sum_{j=\tau}^{j_{2}} 2^{j(1+2 \nu)} \leq C \frac{\ln n_{*}}{n_{*}} 2^{j_{2}(1+2 \nu)} \\
& \leq C\left(\frac{\ln n_{*}}{n_{*}}\right)^{2 s /(2 s+2 \nu+1)} .
\end{aligned}
$$

For $r \geq 1$ and $p \geq 2$, since $B_{p, r}^{s}(M) \subseteq B_{2, \infty}^{s}(M)$, we have

$$
S_{4,2} \leq \sum_{j=j_{2}+1}^{\infty} \sum_{k=0}^{2^{j}-1} \beta_{j, k}^{2} \leq C 2^{-2 j_{2} s} \leq C\left(\frac{\ln n_{*}}{n_{*}}\right)^{2 s /(2 s+2 \nu+1)} .
$$

For $r \geq 1, p \in[1,2)$ and $s>(2 \nu+1) / p$, using $\beta_{j, k}^{2} \mathbf{1}_{\left\{\left|\beta_{j, k}\right|<2 \kappa \lambda_{j}\right\}} \leq C \lambda_{j}^{2-p}\left|\beta_{j, k}\right|^{p}$, $B_{p, r}^{s}(M) \subseteq B_{2, \infty}^{s+1 / 2-1 / p}(M)$ and $(2 s+2 \nu+1)(2-p) / 2+(s+1 / 2-1 / p+\nu-$ $2 \nu / p) p=2 s$, we have

$$
\begin{aligned}
S_{4,2} & \leq C \sum_{j=j_{2}+1}^{j_{1}} \lambda_{j}^{2-p} \sum_{k=0}^{2^{j}-1}\left|\beta_{j, k}\right|^{p} \\
& =C\left(\frac{\ln n_{*}}{n_{*}}\right)^{(2-p) / 2} \sum_{j=j_{2}+1}^{j_{1}} 2^{j \nu(2-p)} \sum_{k=0}^{2^{j}-1}\left|\beta_{j, k}\right|^{p} \\
& \leq C\left(\frac{\ln n_{*}}{n_{*}}\right)^{(2-p) / 2} \sum_{j=j_{2}+1}^{\infty} 2^{j \nu(2-p)} 2^{-j(s+1 / 2-1 / p) p} \\
& \leq C\left(\frac{\ln n_{*}}{n_{*}}\right)^{(2-p) / 2} 2^{-j_{2}(s+1 / 2-1 / p+\nu-2 \nu / p) p} \\
& \leq C\left(\frac{\ln n_{*}}{n_{*}}\right)^{2 s /(2 s+2 \nu+1)} .
\end{aligned}
$$

So, for $r \geq 1,\{p \geq 2$ and $s>0\}$ or $\{p \in[1,2)$ and $s>(2 \nu+1) / p\}$, we have

$$
S_{4} \leq C\left(\frac{\ln n_{*}}{n_{*}}\right)^{2 s /(2 s+2 \nu+1)} .
$$

It follows from (28), (29), (31) and (32) that

$$
S \leq C\left(\frac{\ln n_{*}}{n_{*}}\right)^{2 s /(2 s+2 \nu+1)} .
$$

Combining (25), (26), (27) and (33), we have, for $r \geq 1,\{p \geq 2$ and $s>0\}$ or $\{p \in[1,2)$ and $s>(2 \nu+1) / p\}$,

$$
\mathbb{E}\left(\int_{0}^{1}\left(\widehat{f}^{H}(x)-f_{\sigma^{2}}(x)\right)^{2} d x\right) \leq C\left(\frac{\ln n_{*}}{n_{*}}\right)^{2 s /(2 s+2 \nu+1)} .
$$

The proof of Theorem 2 is complete.

This work is supported by ANR grant NatImages, ANR-08-EMER-009. 


\section{References}

Butucea, C. and Matias, C. (2005). Minimax estimation of the noise level and of the signal density in a semiparametric convolution model. Bernoulli, 11, 2, 309-340.

Cai, T. (1999). Adaptive Wavelet Estimation: A Block Thresholding And Oracle Inequality Approach. The Annals of Statistics, 27, 898-924.

Cai, T. (2002). On adaptive wavelet estimation of a derivative and other related linear inverse problems. Journal of Statistical Planning and Inference, 108, 329-349.

Carrasco, M. and Chen, X., (2002). Mixing and moment properties of various GARCH and stochastic volatility models. Econometric Theory, 18, 17-39.

Caroll, R.J. and Hall, P. (1988). Optimal rates of convergence for deconvolving a density. $J$. Amer. Statist. Assoc., 83, 1184-1186.

Chaubey, Y.P., Chesneau, C. and Doosti, H. (2010). On Linear Wavelet Density Estimation: Some Recent Developments, Journal of the Indian Society of Agricultural Statistics, to appear.

Chaubey, Y.P., Chesneau, C. and Doosti, H. (2010). Adaptive wavelet estimation of a density from mixtures under multiplicative censoring, Preprint, Concordia University.

Chesneau, C., Fadili, J. and Starck, J.-L. (2010). Stein block thresholding for image denoising. Applied and Computational Harmonic Analysis, 28, 1, 67-88.

Cohen, A., Daubechies, I., Jawerth, B. and Vial, P. (1993). Wavelets on the interval and fast wavelet transforms. Applied and Computational Harmonic Analysis, 24, 1, 54-81.

Comte, F., Rozenholc, Y. and Taupin, M.-L. (2006). Penalized contrast estimator for density deconvolution. The Canadian Journal of Statistics, 34, 431-452.

Comte, F., Dedecker, J. and Taupin, M.-L. (2008). Adaptive density estimation for general ARCH models, Econometric Theory, 24, 6, 1628-1662.

Davydov, Y, (1970). The invariance principle for stationary processes. Theor. Probab. Appl., $15,3,498-509$

Delaigle, A. and Gijbels, I. (2006). Estimation of boundary and discontinuity points in deconvolution problems. Statistica Sinica, 16, $773-788$

Delyon, B. and Juditsky, A. (1996). On minimax wavelet estimators, Applied Computational Harmonic Analysis, 3, 215-228.

Devroye, L. (1989). Consistent deconvolution in density estimation. Canad. Journ. Statist., $17,235-239$.

Donoho, D.L., Johnstone, I.M., Kerkyacharian, G. and Picard, D. (1996). Density estimation by wavelet thresholding, The Annals of Statistics, 24, 508-539.

Doukhan, P. (1994). Mixing. Properties and Examples. Lecture Notes in Statistics 85. Springer Verlag, New York.

Fan, J. (1991). On the optimal rates of convergence for nonparametric deconvolution problem. Ann. Statist., 19, 1257-1272.

Fan, J. and Koo, J.Y. (2002). Wavelet deconvolution. IEEE transactions on information theory, 48, 734-747.

Lacour, C. (2006). Rates of convergence for nonparametric deconvolution. C. R. Acad. Sci. Paris Ser. I Math., 342 (11), 877-882.

Masry, E. (1991). Multivariate probability density deconvolution for stationary random processes. IEEE Inf Th, 37, 1105-1115.

Meyer, Y. (1992). Wavelets and Operators. Cambridge University Press, Cambridge.

Modha, D. and Masry, E. (1996). Minimum complexity regression estimation with weakly dependent observations. IEEE Trans. Inform. Theory, 42, 2133-2145.

Pensky, M. and Vidakovic, B. (1999). Adaptive wavelet estimator for nonparametric density deconvolution. The Annals of Statistics, 27, 2033-2053.

Van Zanten, H. and Zareba, P. (2008). A note on wavelet density deconvolution for weakly dependent data. Stat. Inference Stoch. Process., 11, 207-219.

Withers, C. S. (1981). Conditions for linear processes to be strong-mixing. Zeitschrift für Wahrscheinlichkeitstheorie und Verwandte Gebiete, 57, 477-480. 